\title{
Existence of isotropic complete solutions of the П-Hamilton-Jacobi equation
}

\author{
Sergio Grillo \\ Instituto Balseiro, Universidad Nacional de Cuyo and CONICET \\ Av. Bustillo 9500, San Carlos de Bariloche \\ R8402AGP, República Argentina
}

February 7, 2019

\begin{abstract}
Consider a symplectic manifold $M$, a Hamiltonian vector field $X$ and a fibration $\Pi: M \rightarrow N$. Related to these data we have a generalized version of the (time-independent) Hamilton-Jacobi equation: the $\Pi$-HJE for $X$, whose unknown is a section $\sigma: N \rightarrow M$ of $\Pi$. The standard HJE is obtained when the phase space $M$ is a cotangent bundle $T^{*} Q$ (with its canonical symplectic form), $\Pi$ is the canonical projection $\pi_{Q}: T^{*} Q \rightarrow Q$ and the unknown is a closed 1-form $\mathrm{d} W: Q \rightarrow T^{*} Q$. The function $W$ is called Hamilton's characteristic function. Coming back to the generalized version, among the solutions of the $\Pi-\mathrm{HJE}$, a central role is played by the so-called isotropic complete solutions. This is because, if a solution of this kind is known for a given Hamiltonian system, then such a system can be integrated up to quadratures. The purpose of the present paper is to prove that, under mild conditions, an isotropic complete solution exists around almost every point of $M$. Restricted to the standard case, this gives rise to an alternative proof for the local existence of a complete family of Hamilton's characteristic functions.
\end{abstract}

\section{Introduction}

In the recent few years, several extensions of the Hamilton-Jacobi Theory have been developed. See for instance [4, 6, 17, 9, 10]. In Ref. [12, it was presented an extension to general dynamical systems (on fibered phase spaces), which contains as particular cases the previuos ones. Let us briefly review it in the restricted context of Hamiltonian systems. Consider a symplectic manifold $(M, \omega)$ of dimension $d=2 s$, a second manifold $N$ of dimension $k$ and a surjective submersion $\Pi: M \rightarrow N$. Consider also a Hamiltonian system with phase space $M$ and a Hamiltonian function $H$. According to Ref. [12, a (global) complete solution of the so-called П-HJE (П-Hamilton-Jacobi Equation), for the Hamiltonian vector field $X_{H}$, is a surjective local diffeomorphism $\Sigma: N \times \Lambda \rightarrow M$ such that

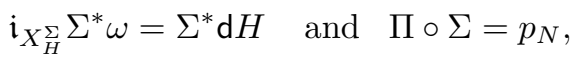

where $\Lambda$ is a third manifold of dimension $d-k$, the vector field $X_{H}^{\Sigma} \in \mathfrak{X}(N \times \Lambda)$ is given by

$$
X_{H}^{\Sigma}(n, \lambda)=\left(\Pi_{*}\left(X_{H}(\Sigma(n, \lambda))\right), 0\right),
$$

and $p_{N}: N \times \Lambda \rightarrow N$ is the canonical projection onto the first factor. Naturally, a local complete solution of the $\Pi$-HJE for $X_{H}$, along an open subset $U$, is a complete solution of the $\left.\Pi\right|_{U}$-HJE for $\left.X_{H}\right|_{U}$. (Here, we are seeing $\left.\Pi\right|_{U}$ as a fibration onto $\Pi(U)$ and $\left.X_{H}\right|_{U}$ as a vector field on $\left.U\right)$. For each $\lambda \in \Lambda$, the function

$$
\sigma_{\lambda}: N \rightarrow M: n \mapsto \Sigma(n, \lambda)
$$


is a section of $\Pi$ and it is called a partial solution (or simply a solution) of the $\Pi$-HJE. On the other hand, $\Sigma$ is said to be isotropic if

$$
\sigma_{\lambda}^{*} \omega=0, \quad \forall \lambda \in \Lambda,
$$

or, equivalently, if each vector subspace

$$
\Sigma_{*}\left(T_{n} N \times\{0\}_{\lambda}\right) \subseteq T_{\Sigma(n, \lambda)} M
$$

is isotropic with respect to $\omega$. Note that, in such a case, we must have $s \geq k$. When $k=s$, above linear spaces are Lagrangian, and consequently $\Sigma$ is said to be Lagrangian.

The standard Hamilton-Jacobi Theory (see for instance [1] and [6]) corresponds to the case in which:

- $M$ is a cotangent bundle $T^{*} Q$,

- $\omega$ is the canonical symplectic form $\omega_{Q}$ of $T^{*} Q$,

- $\Pi$ is the canonical projection $\pi_{Q}: T^{*} Q \rightarrow Q$,

and its complete solutions are precisely the Lagrangian complete solutions of the $\pi_{Q}$-HJE. More precisely (see Ref. [12]), in this case the complete solutions are locally given by the formula $\Sigma(q, \lambda)=\mathrm{d} W_{\lambda}(q)$, where each function $W_{\lambda}$, called Hamilton's characteristic function, satisfies the standard (time-independent) Hamilton-Jacobi equation:

$$
\mathrm{d}\left(H \circ \mathrm{d} W_{\lambda}\right)=0 .
$$

We shall say that the functions $W_{\lambda}$ 's give a complete family of Hamilton's characteristic functions.

The importance of the isotropic complete solutions, as proven in 11, 12 (generalizing a well-known result of the standard theory), lies in that fact that if we know one of them for a given Hamiltonian system, then such a system is exactly solvable. Actually, in order to ensure exact solvability, it is enough to know a local solution (instead of a global one) around every point of the phase space.

It was shown in 12] (see Theorem 4.15 there) that a local complete solution of the П-HJE always exists around every point $m \in M$ such that $X_{H}(m) \notin \operatorname{Ker} \Pi_{*, m}$. However, nothing has been said about the existence of isotropic complete solutions. In this paper we fill in this gap, showing that a local isotropic complete solution does exist around every point $m \in M$ such that, besides the condition $X_{H}(m) \notin \operatorname{Ker} \Pi_{*, m}$, the subspace $\operatorname{Ker} \Pi_{*, m} \subseteq T_{m} M$ is co-isotropic with respect to $\omega$. Moreover, if no fibration is previously fixed, we show that around every point $m$ such that $X_{H}(m) \neq 0$ there always exist a fibration $\pi$ and a local isotropic complete solution of the $\pi$-HJE for $X_{H}$.

It is worth mentioning that our results do not mean that every Hamiltonian system is exactly solvable around such points, because in order to solve its equations of motion is not enough to ensure the existence of an isotropic complete solution, but we need to have a concrete expression of one of it. Thus, such results are mainly of a theoretical nature, and can be interpreted as follows: every Hamiltonian system is "potentially" exactly solvable with the aid of an isotropic complete solution (following the procedure described in Refs. [11, 12]).

The organization of the paper is as follows. In Section 2 we recall the duality between complete solutions and first integrals, because we shall use it to prove our main results. In Section 3.1 we prove the annunciated existence theorem and, as an immediate corollary, we give in Section 3.2 a novel proof for the local existence of a complete family of Hamilton's characteristic functions. As another corollary, and only for completeness, we show in Section 3.3 that every Hamiltonian system has a set of first integrals as those appearing in the definition of a non-commutative or MischenkoFomenko integrable system [17] (see also [13] for a review on the subject), defined around every non-critical point of the Hamiltonian function. In other words, we prove, as a corollary of our main result, that every Hamiltonian system is "potentially" non-commutative integrable around almost every point of its phase space. (It is worth mentioning that 
this fact can also be proven as a direct consequence of the Carathéodory-Jacobi-Lie theorem [15]). Finally, in Section 3.4, using again the above mentioned duality, we show that around every non-critical point of a Hamiltonian system there always exist a fibration and a local isotropic complete solution related to it.

We assume that the reader is familiar with the basic concepts of Differential Geometry (see [5, 14]), and with the basic ideas related to Hamiltonian systems in the context of Symplectic Geometry (see [1, 15, 16]). We shall work in the smooth (i.e. $C^{\infty}$ ) category, focusing exclusively on finite-dimensional smooth manifolds.

\section{The complete solutions - first integrals duality}

To show our main result, we shall use the duality between (isotropic) complete solutions and (isotropic) first integrals stablished in [12. Let us recall such a duality for the case of Hamiltonian systems. Following the same notation as in the Introduction, consider a symplectic manifold $(M, \omega)$ of dimension $d=2 s$, a function $H: M \rightarrow \mathbb{R}$ and a fibration $\Pi: M \rightarrow N$ (i.e. a surjective submersion), with $\operatorname{dim} N=k$.

We shall say that a submersion $F: M \rightarrow \Lambda$ is a first integrals submersion if

$$
\operatorname{Im} X_{H} \subseteq \operatorname{Ker} F_{*} .
$$

We adopt the following convention for defining $X_{H}$ :

$$
\mathrm{i}_{X_{H}} \omega=\mathrm{d} H
$$

Remark. Note that, if $\Lambda=\mathbb{R}^{l}$, the components $f_{1}, \ldots, f_{l}: M \rightarrow \mathbb{R}$ of $F$ define a set of $l$ (functionally) independent first integrals, in the usual sense, for the Hamiltonian system defined by $H$.

Also, we shall say that $F$ is transverse to $\Pi$ if

$$
T M=\operatorname{Ker}_{*} \oplus \operatorname{Ker} F_{*} .
$$

Finally, the map $F$ is said to be isotropic if

$$
\operatorname{Ker} F_{*} \subseteq\left(\operatorname{Ker} F_{*}\right)^{\perp}
$$

where " $\perp$ " indicates the symplectic orthogonal w.r.t. $\omega$. Of course, in such a case $l \geq s$. When $l=s$, above inclusion reduces to an equality and the map $F$ is say to be Lagrangian.

For later convenience, we shall say that an isotropic submersion $F$ is symplectically complete if $\left(\operatorname{Ker} F_{*}\right)^{\perp}$ is an integrable distribution.

It was shown in [12] that, given an isotropic complete solution $\Sigma: N \times \Lambda \rightarrow M$ of the П-HJE for $H$ [see the Eqs. (1.1) and (1.2)], we can construct around every point of $M$ a neighborhood $U$ and a submersion $F: U \rightarrow \Lambda$ such that

- $\left.\operatorname{Im} X_{H}\right|_{U} \subset \operatorname{Ker} F_{*}$ (first integrals),

- $T U=\operatorname{Ker}\left(\left.\Pi\right|_{U}\right)_{*} \oplus \operatorname{Ker} F_{*}$ (transversality),

- $\operatorname{Ker} F_{*} \subseteq\left(\operatorname{Ker} F_{*}\right)^{\perp}$ (isotropy).

In other words, from $\Sigma$ we have, around every point of $M$, a first integrals submersion, transverse to $\Pi$ and isotropic [see Eqs. (2.1), (2.3) and (2.4)]. $U$ and $F$ are given by the formulae

$$
U:=\Sigma(V) \text { and } \quad F:=p_{\Lambda} \circ\left(\left.\Sigma\right|_{V}\right)^{-1}
$$


where $V \subseteq N \times \Lambda$ is an open subset for which $\left.\Sigma\right|_{V}$ is a diffeomorphism with its image and $p_{\Lambda}: N \times \Lambda \rightarrow \Lambda$ is the canonical projection onto the second factor.

Reciprocally (see also [12]), from a submersion $F: M \rightarrow \Lambda$ satisfying (2.1), (2.3) and (2.4), we can construct, around every point of $M$, a neighborhood $U$ and a local isotropic complete solution $\Sigma$ of the П-HJE. The involved subset $U$ is one for which $\left.(\Pi, F)\right|_{U}$ is a diffeomorphism with its image, and $\Sigma$ is given by

$$
\Sigma=\left[\left.(\Pi, F)\right|_{U}\right]^{-1}: \Pi(U) \times F(U) \rightarrow U .
$$

Summarizing, an isotropic complete solution gives rise to local isotropic first integrals via the Eq. (2.5), and isotropic first integrals give rise to a local isotropic complete solution via the Eq. (2.6).

\section{The existence theorems}

Let us continue with a symplectic manifold $(M, \omega)$ of dimension $d=2 s$, a function $H: M \rightarrow \mathbb{R}$ and a fibration $\Pi: M \rightarrow N$ with $\operatorname{dim} N=k \geq 1$. Now let us assume that, for a given $m \in M$,

i. $X_{H}(m) \notin \operatorname{Ker}_{*, m}$ (in particular, $\left.X_{H}(m) \neq 0\right)$;

ii. $\operatorname{Ker}_{*, m}$ is co-isotropic, i.e. $\left(\operatorname{Ker} \Pi_{*, m}\right)^{\perp} \subseteq \operatorname{Ker}_{*, m}$.

Note that, defining $l:=\operatorname{dim} \operatorname{Ker} \Pi_{*, m}=d-k$, condition (ii) implies the inequalities

$$
1 \leq k \leq s \leq l \leq d
$$

The next proposition constitutes the central result of the paper. From it, we shall derive almost all the other results.

Proposition 1. Under above assumptions, the following assertions hold:

1. There exists an open neighborhood $U$ of $m$ and a distribution $\mathcal{D}$ along $U$ such that:

(a) $\left.\operatorname{Im} X_{H}\right|_{U} \subset \mathcal{D}$

(b) $T U=\operatorname{Ker}\left(\left.\Pi\right|_{U}\right)_{*} \oplus \mathcal{D}$,

(c) $\mathcal{D} \subseteq \mathcal{D}^{\perp}$,

(d) $\mathcal{D}$ is integrable. In particular, there exists a submersion $F: U \rightarrow \mathbb{R}^{l}$ such that $\mathcal{D}=\operatorname{Ker} F_{*}$.

2. $\mathcal{D}$ can be chosen such that $\mathcal{D}^{\perp}$ is also integrable.

In other words, a first integrals submersion, transverse to $\Pi$, isotropic and symplectically complete, can be defined around $m$.

Proof. Let us begin with the first assertion. To do that, given $r \in \mathbb{N}$, consider the property $\mathbf{P}_{r}$ : there exists a neighborhood $U_{r}$ of $m$ and Hamiltonian vector fields $X_{1}, \ldots, X_{r} \in \mathfrak{X}\left(U_{r}\right)$ which are linearly independent, mutually orthogonal w.r.t. $\omega$ (and consequently $\left[X_{i}, X_{j}\right]=0$ for all $i, j$ ) 1 with one of them equal to $\left.X_{H}\right|_{U_{r}}$, and such that the distribution $\mathcal{D}_{r}:=\left\langle X_{1}, \ldots, X_{r}\right\rangle$ satisfies

$$
\left.\mathcal{D}_{r}\right|_{p} \cap \operatorname{Ker}_{*, p}=\{0\}, \quad \forall p \in U_{r}
$$

\footnotetext{
${ }^{1}$ Recall that, given to functions $f, g$, for their Hamiltonian vector fields [using convention [2.2] ] we have that

$$
\left[X_{f}, X_{g}\right]=-X_{\omega\left(X_{f}, X_{g}\right)}
$$
}

Then, if $\omega\left(X_{f}, X_{g}\right)=0$, it follows that $\left[X_{f}, X_{g}\right]=0$. 
We shall show by induction the property $\mathbf{P}_{r}$ for all $r \leq k$. From the validity of $\mathbf{P}_{k}$, it is clear that there exists a distribution $\mathcal{D}_{k}$ satisfying the points $(a)$ to $(d)$ above.

If $r=1$, we can take

$$
U_{1}:=\left\{p \in M: X_{H}(p) \notin \operatorname{Ker}_{*, p}\right\}
$$

and define $X_{1}:=\left.X_{H}\right|_{U_{1}}$. Suppose that $k>1$ and that $\mathbf{P}_{r}$ is true for some $r<k$, and let us show that, as a consequence, $\mathbf{P}_{r+1}$ is also true. (Note that $\left.r+1 \leq k\right)$. Fix a subset $U_{r}$ and Hamiltonian vector fields $X_{1}, \ldots, X_{r} \in \mathfrak{X}\left(U_{r}\right)$ ensured by the property $\mathbf{P}_{r}$. Assume for simplicity that $X_{1}=\left.X_{H}\right|_{U_{r}}$. Since the given vector fields satisfy $\left[X_{i}, X_{j}\right]=0$ for all $i, j$ (see the last footnote), there exists a coordinate chart $\left(U_{r+1},\left(y_{1}, \ldots, y_{d}\right)\right)$ such that $m \in U_{r+1} \subseteq U_{r}$ and

$$
X_{1}(p)=\left.\frac{\partial}{\partial y_{1}}\right|_{p}, \ldots, X_{r}(p)=\left.\frac{\partial}{\partial y_{r}}\right|_{p}, \quad \forall p \in U_{r+1}
$$

Assume for simplicity that $U_{r}=U_{r+1}$. Then we can write

$$
X_{1}=\frac{\partial}{\partial y_{1}}, \ldots, X_{r}=\frac{\partial}{\partial y_{r}}
$$

Note that, for the submersion $F_{r}:=\left(y_{r+1}, \ldots, y_{d}\right): U_{r} \rightarrow \mathbb{R}^{d-r}$, we have that [see the point $(d)$ ]

$$
\operatorname{Ker}\left(F_{r}\right)_{*}=\left\langle\frac{\partial}{\partial y_{1}}, \ldots, \frac{\partial}{\partial y_{r}}\right\rangle=\left\langle X_{1}, \ldots, X_{r}\right\rangle=\mathcal{D}_{r}
$$

Now, consider the Hamiltonian vector fields $X_{y_{1}}, \ldots, X_{y_{d}} \in \mathfrak{X}\left(U_{r+1}\right)$. Using the orthogonality property $\omega\left(X_{i}, X_{j}\right)=0$, for all $1 \leq i, j \leq r$, and the fact that

$$
\omega\left(X_{y_{a}}, X_{j}\right)=\left\langle d y_{a}, \frac{\partial}{\partial y_{j}}\right\rangle=\delta_{a j}, \quad 1 \leq a \leq d, \quad 1 \leq j \leq r
$$

if we write $X_{i}$ as a linear combination of the $X_{y_{a}}$ 's, i.e. $X_{i}=\sum_{a=1}^{d} c_{i}^{a} X_{y_{a}}$, we have that $c_{i}^{j}=0$ for all $1 \leq i, j \leq r$. That is to say,

$$
X_{i}=\sum_{a=r+1}^{d} c_{i}^{a} X_{y_{a}}
$$

Note that, since $r<k \leq d / 2$, we have that $d-r>r$. Then, since the matrix with coefficients $c_{i}^{a}$ 's (with $i=1, \ldots, r$ and $a=r+1, \ldots, d)$ must have maximal rank $r$ (because the $X_{i}$ 's are l.i.), we can reorder the $d-r$ coordinates $y_{a}$ 's (with $a$ between $r+1$ and $d$ ) to ensure that the last $r$ columns define an invertible matrix. For this new order, it is easy to see that the set of vector fields

$$
\left\{X_{1}, \ldots, X_{r}, X_{y_{1}}, \ldots, X_{y_{r}}, X_{y_{r+1}}, \ldots, X_{y_{d-r}}\right\}
$$

gives a basis for $T U_{r+1}$. Let us focus on the fields above evaluated at $m$. We shall see that there exists a natural number $b$ between $r+1$ and $d-r$ such that

$$
\left\{X_{1}(m), \ldots, X_{r}(m), X_{y_{b}}(m)\right\} \cap \operatorname{Ker}_{*, m}=\{0\}
$$

This would say that the Hamiltonian vector fields $X_{1}, \ldots, X_{r}, X_{r+1}$, with $X_{r+1}=X_{y_{b}}$, are independent, mutually orthogonal, with one of them equal to $\left.X_{H}\right|_{U_{r+1}}$, and such that the distribution generated by them $\mathcal{D}_{r+1}:=\left\langle X_{1}, \ldots, X_{r+1}\right\rangle$ satisfies

$$
\left.\mathcal{D}_{r+1}\right|_{p} \cap \operatorname{Ker}_{*, p}=\{0\}, \quad \forall p \in U_{r+1},
$$

shrinking $U_{r+1}$ if necessary [since (3.6) is an open condition]. In other words, we would prove that $\mathbf{P}_{r+1}$ is true. 
In order to show the validity of (3.6), we shall proceed by reductio ad absurdum. Suppose first that $r<k / 2$. If (3.6) does not hold for any $b$ between $r+1$ and $d-r$, then there exist numbers $A_{b}^{j}, \alpha_{b} \in \mathbb{R}$ such that

$$
0 \neq u_{b}:=\sum_{j=1}^{r} A_{b}^{j} X_{j}(m)+\alpha_{b} X_{y_{b}}(m) \in \mathrm{Ker}_{*, m}, \quad b=r+1, \ldots, d-r
$$

If $\sum_{j=1}^{r} A_{b}^{j} X_{j}(m)=0$, then $\alpha_{b} \neq 0$ (to ensure that $u_{b} \neq 0$ ), and if $\sum_{j=1}^{r} A_{b}^{j} X_{j}(m) \neq 0$, the inductive hypothesis ensures that $\sum_{j=1}^{r} A_{b}^{j} X_{j}(m)$ is outside $\operatorname{Ker}_{*, m}$, what forces again to have $\alpha_{b} \neq 0$. Hence, the vectors $u_{b}$ 's define a set of

$$
(d-r)-(r+1)+1=d-2 r
$$

linearly independent vectors inside de $\operatorname{Ker}_{*, m}$. But

$$
d-2 r>d-k=l=\operatorname{dim} \operatorname{Ker}_{*, m}
$$

(because we are assuming that $2 r<k$ ), which is a contradiction. Now, let us consider the complementary case: $k / 2 \leq r<$ $k$. Again, if (3.6) does not hold for any $b$ between $r+1$ and $d-r$, we have the $d-2 r$ independent vectors $u_{b} \in \mathrm{Ker}_{*, m}$ as above [see (3.7)]. Since now

$$
d-2 r \leq d-k=l
$$

to complete a basis for $\operatorname{Ker}_{*, m}$ we can add $l-(d-2 r)=2 r-k$ additional vectors to the $u_{b}$ 's. If we call them $\left\{v_{1}, \ldots, v_{2 r-k}\right\}$, the set

$$
\left\{u_{r+1}, \ldots, u_{d-r}, v_{1}, \ldots, v_{2 r-k}\right\}
$$

is a basis for $\operatorname{Ker}_{*, m}$. Note that, since $r<k$, then

$$
2 r-k<r
$$

Let us show that there exists a non-null vector $\left.v \in \mathcal{D}_{r}\right|_{m}$ such that

$$
\omega\left(v, v_{a}\right)=0, \quad 1 \leq a \leq 2 r-k
$$

Writing $v=\sum_{i=1}^{r} x_{i} X_{i}(m)$ and, using Eq. (3.5),

$$
v_{a}=\sum_{i=1}^{r} y_{i} X_{i}(m)+\sum_{j=1}^{d-r} B_{a}^{j} X_{y_{j}}(m)
$$

Eq. (3.9) translates to [see Eq. (3.3) and recall that $d-r>r$ ]

$$
\mathbf{B} \cdot\left(\begin{array}{c}
x_{1} \\
x_{2} \\
\vdots \\
x_{r}
\end{array}\right)=\mathbf{0} .
$$

Since $\mathbf{B}$ is a rectangular $(2 r-k) \times r$ matrix, with more columns than rows [recall Eq. (3.8)], the above linear system has a non-trivial solution, as we wanted to show. Since in addition [see (3.3) and (3.7)]

$$
\omega\left(v, u_{a}\right)=0, \quad r+1 \leq a \leq d-r
$$

it follows that $v$ belongs to $\left(\mathrm{Ker}_{*, m}\right)^{\perp}$. But $\mathrm{Ker}_{*, m}$ is co-isotropic, what implies that $v \in \mathrm{Ker} \Pi_{*, m}$. So, $v \neq 0$ and $\left.v \in \mathcal{D}_{r}\right|_{m} \cap \operatorname{Ker}_{*, m}$, which is a contradiction. As a consequence, for any $r<k$, there always exists $b$ fulfilling Eq. (3.6), as claimed. 
Summarizing, if $r+1=k$, calculations above show that the neighborhood $U:=U_{k}$ of $m$ and the distribution $\mathcal{D}:=\left\langle X_{1}, \ldots, X_{k}\right\rangle$ along $U$ satisfy the points $a, b, c$ and $d$. It rests to show the last assertion. To do that, fix a coordinate chart $\left(U,\left(y_{1}, \ldots, y_{d}\right)\right)$ (shrinking $U$ if needed) for which (3.2) holds (for $r=k$ ). Note that, according to (3.3),

$$
X_{1}, \ldots, X_{k}, X_{y_{k+1}}, \ldots, X_{y_{d-k}} \in \mathcal{D}^{\perp}
$$

and according to Eq. (3.4) and the discussion below, we can reorder the coordinates such that above $d-k$ vector fields are linearly independent. Finally, since $\operatorname{dim} \mathcal{D}^{\perp}=d-k$, it follows that

$$
\left\langle X_{1}, \ldots, X_{k}, X_{y_{k+1}}, \ldots, X_{y_{d-k}}\right\rangle=\mathcal{D}^{\perp}
$$

So, we just need to show for the vector fields $X_{y_{a}}, X_{y_{b}} \in \mathcal{D}^{\perp}$ that $\left[X_{y_{a}}, X_{y_{b}}\right] \in \mathcal{D}^{\perp}$ (because for the other pairs of vector fields this follows from orthogonality). On the one hand, we have that

$$
\omega\left(\left[X_{y_{a}}, X_{y_{b}}\right], X_{i}\right)=-\left\{\left\{y_{a}, y_{b}\right\}, f_{i}\right\}=\left\{\left\{f_{i}, y_{a}\right\}, y_{b}\right\}+\left\{\left\{y_{b}, f_{i}\right\}, y_{a}\right\},
$$

being $f_{i}$ the Hamiltonian of $X_{i}$ and $\{\cdot, \cdot\}$ the Poisson bracket associated to $\omega$. On the other hand, using (3.3), $\left\{f_{i}, y_{a}\right\}=$ $\omega\left(X_{i}, X_{y_{a}}\right)=0$ (for $1 \leq i \leq k$ and $k+1 \leq a \leq d-k$ ). Consequently $\left[X_{y_{a}}, X_{y_{b}}\right] \in \mathcal{D}^{\perp}$, which implies that $\mathcal{D}^{\perp}$ is integrable.

\subsection{Isotropic complete solutions of the П-HJE}

Using above proposition and the duality between first integrals and complete solutions, the main result of this paper immediately follows.

Theorem 1. Consider a symplectic manifold $(M, \omega)$, a Hamiltonian vector field $X_{H} \in \mathfrak{X}(M)$ and a fibration $\Pi: M \rightarrow N$. Assume that $X_{H}(m) \notin \operatorname{Ker}_{*, m}$ and $\operatorname{Ker}_{*, m}$ is co-isotropic for some point $m \in M$. Then, there exists a neighborhood $U$ of $m$ and a local isotropic complete solution of the $\Pi$-HJE for $X_{H}$ along $U$.

Proof. From proposition above we know that there exists a neighborhood $U$ of $m$ and a submersion $F: U \rightarrow \mathbb{R}^{l}$ satisfying (2.1), (2.3) (replacing $M$ by $U$ and $\Pi$ by $\left.\Pi\right|_{U}$ ) and (2.4). It is clear from the transversality property (2.3) that $U$ can be taken such that $\left(\left.\Pi\right|_{U}, F\right)$ is a diffeomorphism. Then, as we said in Section 2 [see Eq. (2.6)], the inverse of $\left(\left.\Pi\right|_{U}, F\right): U \rightarrow \Pi(U) \times F(U)$ is a local isotropic complete solution of the $\Pi$-HJE for $X_{H}$ along $U$.

Remark. Note that the second point of Proposition 1 is not used in above theorem. It will be used in Section 3.3 ,

In the following, we shall present some other consequences of Proposition (11). For instance, if no fibration is fixed beforehand, we shall see in Section 3.4 that an isotropic complete solution exists around $m$ for some fibration $\pi$, with the only condition that $X_{H}(m) \neq 0$ (i.e. $m$ is not a critical point for $X_{H}$ ).

\subsection{Standard complete solutions}

Suppose that we are in the standard situation, i.e. $M=T^{*} Q, \omega=\omega_{Q}, N=Q$ and $\Pi=\pi_{Q}$. Observe that, following above notation, we have that $\operatorname{dim} Q=s=k$. It is well-known that $\operatorname{Ker}\left(\pi_{Q}\right)_{*, m} \subseteq T_{m} T^{*} Q$ is Lagrangian for all $m \in T^{*} Q$ (and consequently co-isotropic) with respect to $\omega_{Q}$. In fact, fixing a Darboux coordinates chart $\left(U, \varphi=\left(q^{1}, \ldots, q^{s}, p_{1}, \ldots, p_{s}\right)\right)$ around $m$, since

$$
\operatorname{Ker}\left(\pi_{Q}\right)_{*, m}=\left\langle\left.\frac{\partial}{\partial p_{1}}\right|_{m}, \ldots,\left.\frac{\partial}{\partial p_{s}}\right|_{m}\right\rangle
$$

the identity

$$
\operatorname{Ker}\left(\pi_{Q}\right)_{*}=\left(\operatorname{Ker}\left(\pi_{Q}\right)_{*}\right)^{\perp}
$$


is immediate. Then, if $X_{H}(m) \notin \operatorname{Ker}\left(\pi_{Q}\right)_{*, m}$, above theorem ensures that a local isotropic complete solution exists around $m$. But, since $k=s$, such a solution is actually Lagrangian. As explained in the Introduction (see [12] for more details), the Lagrangian complete solutions of the $\pi_{Q}$-HJE for $H$ are locally given by the expression

$$
\Sigma(q, \lambda)=\mathrm{d} W_{\lambda}(q)
$$

with each function $W_{\lambda}$ satisfying (1.3). All that can be condensed in the next result.

Corollary 1. Given an s-manifold $Q$ and a Hamiltonian function $H: T^{*} Q \rightarrow \mathbb{R}$, around every point $m \in T^{*} Q$ such that $X_{H}(m) \notin \operatorname{Ker}\left(\pi_{Q}\right)_{*, m}$ there exists a neighborhood $U \subseteq T^{*} Q$ of $m$, another s-manifold $\Lambda$ and a family of functions $W_{\lambda}: \pi_{Q}(U) \rightarrow \mathbb{R}$ such that

$$
\mathrm{d}\left(H \circ \mathrm{d} W_{\lambda}\right)=0, \quad \forall \lambda \in \Lambda,
$$

and

$$
(q, \lambda) \in \pi_{Q}(U) \times \Lambda \longmapsto \mathrm{d} W_{\lambda}(q) \in U
$$

is a diffeomorphism. In other terms, under above conditions, a complete family of Hamilton's characteristic functions exists around $m$.

Just to analyze a particular situation, but a very common one, suppose that $H$ is of the form

$$
H=\frac{1}{2} \mathfrak{H}+h \circ \pi_{Q},
$$

where $\mathfrak{H}: T^{*} Q \rightarrow \mathbb{R}$ is a quadratic form defined by a Riemannian metric $\phi: T Q \times_{Q} T Q \rightarrow \mathbb{R}$ on $Q$, i.e.

$$
\mathfrak{H}(m)=\phi\left(\phi^{\sharp}(m), \phi^{\sharp}(m)\right),
$$

and $h \in C^{\infty}(Q)$. In other words, suppose that $H$ is simple. Then, as it is easy to show,

$$
\left(\pi_{Q}\right)_{*}\left(X_{H}(m)\right)=\phi^{\sharp}(m),
$$

what implies that $X_{H}(m) \in \operatorname{Ker}\left(\pi_{Q}\right)_{*, m}$ if and only if $m=0$ (i.e. $m$ belongs to the null subbundle of $T^{*} Q$ ). As a consequence, we have the following corollary.

Corollary 2. Consider a manifold $Q$ and a simple Hamiltonian function $H: T^{*} Q \rightarrow \mathbb{R}$. Then, if $m \neq 0$, a complete family of Hamilton's characteristic functions exists around $m$.

\subsection{Non-commutative integrability}

This section is included just for completeness. Let us fix again a symplectic manifold $(M, \omega)$ with $\operatorname{dim} M=2 s=d$.

Definition. Consider a function $H \in C^{\infty}(M)$ and a submersion $F: M \rightarrow \Lambda$. We shall say that the pair $(H, F)$ is a non-commutative or Mischenko-Fomenko integrable system [17] if $F$ is a first integrals submersion, isotropic and symplectically complete. And we shall say that $(H, F)$ is a commutative or Arnold-Liouville integrable system [2] if in addition $F$ is Lagrangian.

As it is well-known, if $(H, F)$ is a non-commutative integrable system, then the Hamiltonian system defined by $H$ can be integrated up to quadratures.

Remark. Usually, additional conditions are asked to $F$, as the compactness and connectedness of their level submanifolds. In such a case, beside the integrability up to quadratures, we can ensure (without any calculation) that the trajectories of the system will be given by quasi-periodic orbits (see [13] for a review on the subject). But we shall not consider in this paper these additional conditions. 
We shall show in this section that, around every non-critical point of $H$, a local submersion $F: U \rightarrow \mathbb{R}^{l}$ always exists such that $\left(\left.H\right|_{U}, F\right)$ is non-commutative integrable. We could do it by following a similar procedure to that applied in the proof of the Proposition 1 Since now no fibration is involved, we can consider properties like $\mathbf{P}_{r}$, but where condition (3.1) is not asked. Also, as in the Appendix of Ref. [11, to prove that result we can use the Carathéodory-Jacobi-Lie theorem [15] (see also the extension given in [8]). Nevertheless, to take advantage of Proposition (10), in this paper we choose to prove it as a corollary of such a proposition, with the aid of the result below.

Proposition 2. Given a (non-necessarily Hamiltonian) vector field $X$, a point $m$ such that $X(m) \neq 0$, and a natural number $k \leq s$, there exist an open neighborhood $U$ of $m$ and a submersion $\pi: U \rightarrow \mathbb{R}^{k}$ such that $X(m) \notin \operatorname{Ker} \pi_{*, m}$ and $\left(\operatorname{Ker} \pi_{*, m}\right)^{\perp} \subseteq \operatorname{Ker} \pi_{*, m}$.

Proof. Let $\left(U,\left(q^{1}, \ldots, q^{s}, p_{1}, \ldots, p_{s}\right)\right)$ be a Darboux coordinate chart around $m$, and let us write $X(m)$ as a linear combination of the related coordinate vector fields, i.e.

$$
X(m)=\left.\sum_{i=1}^{s} a^{i} \frac{\partial}{\partial q^{i}}\right|_{m}+\left.\sum_{j=1}^{s} b_{j} \frac{\partial}{\partial p_{j}}\right|_{m} .
$$

Since $X(m) \neq 0$, then

1. $a^{i} \neq 0$ for some $i$ or

2. $b_{j} \neq 0$ for some $j$.

It is clear that the second case reduces to the first one if we make the canonical transformation

$$
\left(q^{1}, \ldots, q^{s}, p_{1}, \ldots, p_{s}\right) \mapsto\left(-p_{1}, \ldots,-p_{s}, q^{1}, \ldots, q^{s}\right) .
$$

So, we can assume that we are in the first case. In such a case, reordering the coordinates $q^{i}$,s, if needed (and making the same reordering for the coordinates $p_{i}$ 's), we can always assume that $a^{1} \neq 0$. This means that

$$
\left\{X(m),\left.\frac{\partial}{\partial q^{2}}\right|_{m}, \ldots,\left.\frac{\partial}{\partial q^{s}}\right|_{m},\left.\frac{\partial}{\partial p_{1}}\right|_{m}, \ldots,\left.\frac{\partial}{\partial p_{s}}\right|_{m}\right\}
$$

is a basis for $T_{m} M$. Consider now the submersion $\pi:=\left(q_{1}, \ldots, q_{k}\right): U \rightarrow \mathbb{R}^{k}$. Since

$$
\operatorname{Ker} \pi_{*}=\left\langle\frac{\partial}{\partial q^{k+1}}, \ldots, \frac{\partial}{\partial q^{s}}, \frac{\partial}{\partial p_{1}}, \ldots, \frac{\partial}{\partial p_{s}}\right\rangle
$$

and

$$
\left(\operatorname{Ker} \pi_{*}\right)^{\perp}=\left\langle\frac{\partial}{\partial p_{1}}, \ldots, \frac{\partial}{\partial p_{k}}\right\rangle
$$

it is clear that $X(m) \notin \operatorname{Ker} \pi_{*, m}$ and $\operatorname{Ker} \pi_{*}$ is co-isotropic. Thus, $\pi$ is the submersion we are looking for. $\triangle$

Combining Propositions 1 and 2, we have the wanted result.

Theorem 2. Consider a $2 s$-dimensional symplectic manifold $(M, \omega)$ and a function $H \in C^{\infty}(M)$. Assume that, for a given point $m \in M, \mathrm{~d} H(m) \neq 0$. Then, for every $l$ such that $2 s>l \geq s$, there exists a neighborhood $U$ of $m$ and a submersion $F: U \rightarrow \mathbb{R}^{l}$ such that the pair $\left(\left.H\right|_{U}, F\right)$ is a non-commutative integrable system. In particular, choosing $l=s$, we have that $\left(\left.H\right|_{U}, F\right)$ is a commutative integrable system.

Proof. If $\mathrm{d} H(m) \neq 0$, then $X_{H}(m) \neq 0$, and consequently, using Proposition 2 for each $k \leq s$ there exists a fibration $\pi: U \rightarrow \pi(U) \subseteq \mathbb{R}^{k}$ such that $X_{H}(m) \notin \operatorname{Ker} \pi_{*, m}$ and $\operatorname{Ker} \pi_{*, m}$ is co-isotropic. In this situation, given $l$ such that $2 s>l \geq s$, and taking $k=2 s-l$, Proposition 1 ensures the existence of a submersion $F: U \rightarrow \mathbb{R}^{l}$ (shrinking $U$ if needed) satisfying precisely the conditions of the non-commutative integrability (see 1. $(d)$ and then 1. (a), 1. (c) and 2). 


\subsection{Isotropic complete solutions for some fibration}

If no fibration is fixed beforehand, we have the next result.

Theorem 3. Consider a $2 s$-dimensional symplectic manifold $(M, \omega)$ and a function $H \in C^{\infty}(M)$. Assume that, for a given point $m \in M, \mathrm{~d} H(m) \neq 0$. Then, for every $k \leq s$, there exists a neighborhood $U$ of $m$, a fibration $\pi: U \rightarrow \pi(U) \subseteq$ $\mathbb{R}^{k}$ and an isotropic complete solution of the $\pi$-HJE for $\left.X_{H}\right|_{U}$.

Proof. According to Proposition 2, there exist an open neighborhood $U$ and fibration a $\pi: U \rightarrow \pi(U) \subseteq \mathbb{R}^{k}$ such that $X_{H}(m) \notin \operatorname{Ker} \pi_{*, m}$ and $\left(\operatorname{Ker} \pi_{*, m}\right)^{\perp} \subseteq \operatorname{Ker} \pi_{*, m}$. On the other hand, Proposition 1 ensures the existence of another fibration $F: U \rightarrow F(U) \subseteq \mathbb{R}^{l}$ (shrinking $U$ if needed), with $l=d-k$, such that:

- $\left.\operatorname{Im} X_{H}\right|_{U} \subset \operatorname{Ker} F_{*}$,

- $T U=\operatorname{Ker} \pi_{*} \oplus \operatorname{Ker} F_{*}$,

- $\operatorname{Ker} F_{*} \subseteq\left(\operatorname{Ker} F_{*}\right)^{\perp}$.

Then, by duality, assuming for simplicity that $(\pi, F): U \rightarrow \pi(U) \times F(U)$ is a diffeomorphism (otherwise it is enough to change $U$ by a smaller open set), we know that $\Sigma:=(\pi, F)^{-1}$ is an isotropic complete solution of the $\pi$-HJE for $\left.X_{H}\right|_{U}$. $\triangle$

Concluding, around every non-critical point of a Hamiltonian system there exists:

- a submersion $F: U \rightarrow \mathbb{R}^{l}$ such that $\left(\left.H\right|_{U}, F\right)$ is non-commutative integrable (see Theorem 2);

- an isotropic complete solution $\Sigma$ of the $\pi$-HJE for some fibration $\pi: U \rightarrow \pi(U)$ (see Theorem 3).

As we said at the beginning of the paper, above existence results do not mean that, around a non-critical point, every Hamiltonian system is exactly solvable. The point is that, in order to ensure exact solvability, it is not enough to know that the mentioned objects exist, but we need to have an explicit expression of them.

\section{Acknowledgements}

The author thanks CONICET for its financial support.

\section{References}

[1] R. Abraham, J.E. Marsden, Foundation of Mechanics, New York, Benjaming Cummings (1985).

[2] V.I. Arnold, Mathematical Models in Classical Mechanics, Berlin, Springer-Verlag (1978).

[3] V. I. Arnold, V.V. Kozlov and A. I. Neishtadt, Mathematical aspects of classical and celestial mechanics, Itogi Nauki i Tekhniki. Sovr. Probl. Mat. Fundamental'nye Napravleniya, Vol. 3, VINITI, Moscow 1985. English transl.: Encyclopadia of Math. Sciences, Vol.3, Springer- Verlag, Berlin (1989).

[4] P. Balseiro, J.C. Marrero, D. Martín de Diego, E. Padrón, A unified framework for Mechanics, Hamilton-Jacobi equation and applications, Nonlinearity, 23, 8 (2010), 1887-1918.

[5] W.M. Boothby, An Introduction to Differentiable Manifolds and Riemannian Geometry, New York, Academic Press (1985). 
[6] J. Cariñena, X. Gràcia, G. Marmo, E. Martínez, M. Muñoz-Lecanda, N. Roman-Roy, Geometric Hamilton-Jacobi theory, International Journal of Geometric Methods in Modern Physics 3, 7 (2006), 1417-1458.

[7] J. Cariñena, X. Gràcia, G. Marmo, E. Martínez, M. Muñoz-Lecanda, N. Roman-Roy, Geometric Hamilton-Jacobi theory for nonholonomic dynamical systems, International Journal of Geometric Methods in Modern Physics 7, 3 (2010), 431-454.

[8] Camille Laurent-Gengoux, Eva Miranda, Pol Vanhaecke, Action-angle Coordinates for Integrable Systems on Poisson Manifolds, International Mathematics Research Notices, Volume 2011, Issue 8, 1 January 2011, 1839-1869.

[9] M. de León, J.C. Marrero, D. Martín de Diego: Linear almost Poisson structures and Hamilton-Jacobi equation. Applications to nonholonomic Mechanics, J. Geom. Mech. 2 (2010) 159-198.

[10] M. de León, D. Martín de Diego, M. Vaquero: A Hamilton-Jacobi theory on Poisson manifolds, Journal of Geometric Mechanics 6, 1 (2014) 121-140.

[11] S. Grillo, Non-commutative integrability, exact solvability and the Hamilton-Jacobi theory, arXiv:1804.10958v2.

[12] S. Grillo, E. Padrón, A Hamilton-Jacobi Theory for general dynamical systems and integrability by quadratures in symplectic and Poisson manifolds, J. Geom. Phys. 110 (2016), 101-129.

[13] B. Jovanovic, Symmetries and integrability, Publications de L'Institut Mathématique, Nouvelle série 84, 98 (2008), $1-36$.

[14] S. Kobayashi, K. Nomizu, Foundations of Differential Geometry, New York, John Wiley \& Son (1963).

[15] P. Liberman, C.-M. Marle, Symplectic geometry and analytical mechanics, Vol. 35 of Mathematics and its Applications, D. Reidel Publishing Co., Dordrecht 1987.

[16] J.E. Marsden, T.S. Ratiu, Introduction to Mechanics and Symmetry, New York, Springer-Verlag (1994).

[17] A.S. Mischenko, A.T. Fomenko: Generalized Liouville Methods of integration of Hamiltonian systems, Funct. Anal. Appl., 12, 2 (1978), 113-1978. 